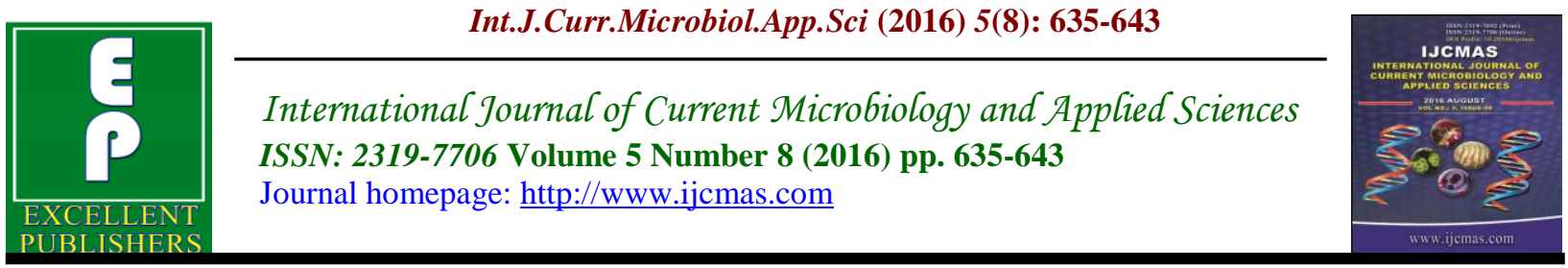

Original Research Article

http://dx.doi.org/10.20546/ijcmas.2016.508.071

\title{
Prevalence and Antibiotic Susceptibility Pattern of Multi-drug Resistant Klebsiella pneumoniae in Pus samples from Surgical Ward Patients in a Tertiary Care Hospital in South India
}

\author{
Md. Mudassar Iqbal Lodhi ${ }^{1} *$ and Shobha D. Nadagir ${ }^{2}$ \\ ${ }^{1}$ Department of Microbiology, ICARE Institute of Medical Sciences and Research and \\ Dr. B. C. Roy Hospital, Haldia, India \\ ${ }^{2}$ Department of Microbiology, Navodaya Medical College Hospital and Research \\ Centre, Raichur, India \\ *Corresponding author
}

Keywords

ESBL,

$\mathrm{AmpC}$,

MBL,

KPC,

Klebsiella pneumoniae.

\begin{tabular}{l}
\hline Article Info \\
\hline Accepted: \\
23 July 2016 \\
Available Online: \\
10 August 2016
\end{tabular}

A B S T R A C T

Klebsiella pneumoniae, being prevalent in the nosocomial environment, is capable of expressing a variety of resistance enzymes, thus justifying their continued monitoring. The present study was undertaken to determine the prevalence of multi-drug resistant Klebsiella pneumoniae isolated from post surgical wound infection and to know their resistance pattern. Consecutive, non-repeat Klebsiella pneumoniae isolates obtained from post surgical wound infection were processed by conventional culture method and antimicrobial susceptibility test. ESBL, AmpC, MBL and KPC production was detected by Phenotypic confirmatory disk diffusion test, AmpC Disk test, Imipenem+EDTA Combined disk test and Modified Hodge Test respectively. Out of 84 pus samples, 67 (79.7\%) were ESBL producers, $36(42.8 \%)$ were AmpC producers and 12 (14.3\%) were MBL producers. Thirty one (36.9\%) Klebsiella pneumoniae isolates were both ESBL and AmpC co-producers whereas ESBL, AmpC and MBL co-producing isolates were $12(14.3 \%)$. However none of the isolates were positive for Klebsiella pneumoniae Carbapenemase (KPC) by Modified Hodge Test. ESBL, AmpC and MBL positive isolates were more drug resistant compared to non-producers for all the antibiotics used in the study. Enhanced infection control, coupled with antibiotic stewardship programs, therefore play an important role in limiting the spread of this terrifying antimicrobial resistance.

\section{Introduction}

Klebsiella is an important human pathogen that has been associated in recent decades with nosocomial outbreaks. Nosocomial Klebsiella infections are mainly caused by Klebsiella pneumoniae, the medically most important species of the genus.
Klebsiella accounts for 3 to $7 \%$ of all nosocomial bacterial infections, placing them among the eight most important infectious pathogens in hospitals (Podschun and Ullmann, 1998). 
Klebsiella pneumoniae strains have the potential to cause severe morbidity and mortality particularly in intensive care units, paediatric and surgical wards (Lim et al., 2009). The common cause of bacterial resistance to beta-lactam antibiotics is the production of ESBL. These transmissible $\beta$ lactamases are plasmid encoded that can be exchanged between bacteria and are inhibited by clavulanic acid, tazobactam and / or sulbactam (Varaiya et al., 2008).

AmpC beta-lactamases are cephalosporinases that are poorly inhibited by clavulanic acid. They can be differentiated from other ESBLs by their ability to hydrolyse cephamycins as well as other extended spectrum cephalosporins. Failure to detect these enzymes has contributed to their uncontrolled spread and to therapeutic failures (Singhal et al., 2005).

Carbapenems play an important role in the treatment of serious infections caused by these pathogens. The beta-lactamasemediated Carbapenem resistance among Klebsiella pneumoniae (KPC) isolates is an emerging problem (Meyer et al., 1993). Increasingly frequent reports of nosocomial outbreaks caused by Klebsiella pneumoniae justify their monitoring in the hospital.

\section{Materials and Methods}

The present study was carried out in the Department of Microbiology, Karnataka Institute of Medical Sciences (KIMS), Hubli from December 2012 to November 2013.

\section{Inclusion criteria}

Consecutive, non-repeat Klebsiella pneumoniae isolates obtained from post surgical wound infection were included in the study.

\section{Exclusion criteria}

Samples obtained from patients with preexisting infection.

Other species namely Klebsiella oxytoca, Klebsiella ornithinolytica, Klebsiella rhinoscleromatis were not included in the study.

Pus samples were collected using a sterile swab under strict aseptic precautions and transported to the laboratory within two hours of collection. They were inoculated onto Chocolate agar and MacConkey agar plates. The plates were incubated aerobically overnight at $37^{\circ} \mathrm{C}$ and observed for growth on the next day. Klebsiella pneumoniae was identified by conventional method based on the morphological characteristics of the colonies and biochemical reactions.

Antibiotic susceptibility testing was done on Mueller-Hinton agar by the Kirby-Bauer disk diffusion method as per CLSI guidelines (CLSI, 2013). Commercially available antibiotic disks obtained from Himedia Laboratories Private Limited Mumbai were used. The panel of antibiotic discs used were Amikacin $(30 \mu \mathrm{g})$, Cefazolin (30 $\mu \mathrm{g})$, Cefepime $(30 \mu \mathrm{g})$, Cefotaxime (30 $\mu \mathrm{g})$, Cefoxitin $(30 \mu \mathrm{g})$, Ceftazidime $(30 \mu \mathrm{g})$, Ceftazidime + Clavulanic acid $(30 / 10 \mu \mathrm{g})$, Ceftriaxone $(30 \mu \mathrm{g})$, Ciprofloxacin $(5 \mu \mathrm{g})$, Cotrimoxazole $(1.25$ / $23.75 \mu \mathrm{g})$, Ertapenem $(10 \mu \mathrm{g})$, Gentamicin $(10 \mu \mathrm{g})$, Imipenem (10 $\mu \mathrm{g})$, Levofloxacin $(5 \mu \mathrm{g})$, Piperacillintazobactam $(100 / 10 \mu \mathrm{g})$ and Tetracycline- 30 $\mu \mathrm{g}$.

Screening test for ESBL production was done using Ceftazidime disks. A zone diameter of $\leq 22 \mathrm{~mm}$ was considered as probable ESBL producer, which was confirmed by Phenotypic confirmatory disc diffusion test using Ceftazidime and Ceftazidime + Clavulanic acid (CLSI, 2013). 
Isolates were screened for $\mathrm{AmpC}$ production using Cefoxitin disk. Isolates with Cefoxitin zone of $<18 \mathrm{~mm}$ were considered as screen positives. Phenotypic confirmation of AmpC beta-lactamase was done by using AmpC disk test (Singhal et al., 2005).

Screening test for MBL production was done using Imipenem disk. Isolates with zone of < $19 \mathrm{~mm}$ were considered as screen positive and were subjected to Imipenem-EDTA Combined disk test for Phenotypic confirmation (Behera et al., 2008).

Isolates were screened for KPC production using Ertapenem disk. Isolates with Ertapenem zones $\leq 21 \mathrm{~mm}$ were considered as screen positive. Phenotypic confirmatory test for KPC production was done using Modified Hodge Test (MHT) (CLSI, 2013).

\section{Statistical analysis}

Statistical analysis was done by Chi-square test using SPSS software and test of proportions wherever applicable.

\section{Results and Discussion}

Of the consecutive, non-repeat 200 pus samples received from surgical wards, 84 (42\%) were Klebsiella pneumoniae isolates. Of these, 67 (79.7\%) were ESBL producers, $36(42.8 \%)$ were AmpC producers and 12 $(14.3 \%)$ were MBL producers. Thirty one (36.9\%) Klebsiella pneumoniae isolates were both ESBL and AmpC co-producers whereas ESBL, AmpC and MBL coproducing isolates were 12 (14.3\%). However none of the isolates were positive for Klebsiella pneumoniae Carbapenemase (KPC) by Modified Hodge Test.

Maximum number of ESBL and AmpC producing isolates, were found in the age group of 21-30 years whereas that of MBL producing isolates, were found in the age group of 31-40 years. Predominantly affected gender was male, with $56.6 \%$, $63.9 \%$ and $73.7 \%$ respectively for ESBL, Amp C and MBL producers.

Antibiotic resistance pattern of ESBL, Amp $\mathrm{C}$ and MBL producing isolates are presented in Table No 1. Compared to non betalactamase producers, difference in drug resistance was statistically significant for all the antibiotics used in the study.

Klebsiella pneumoniae is a Gram negative bacillus often involved in severe community-acquired and nosocomial infections. Its distribution as a pathogen is uneven across the world with the highest incidence in developing countries and in Asia than in Europe and the United States. A critical feature of Klebsiella pneumoniae as a successful healthcare-associated pathogen has been its evolution into a multi drug resistant (MDR) Gram-negative bacillus. MDR strains of Klebsiella pneumoniae have caused a number of nosocomial infection outbreaks in Intensive Care Units (ICU) and neonatal nurseries. It primarily attacks immunocompromised individuals who are hospitalized and suffer from severe underlying diseases such as diabetes mellitus and chronic pulmonary obstruction (Harrison's principles of Internal Medicine, $18^{\text {th }}$ edition).

The most common cause of bacterial resistance to beta-lactam antibiotics is the production of beta-lactamases. Extended Spectrum Beta-Lactamases (ESBL) represent a major group of betalactamases currently being identified worldwide in large numbers along with inducible AmpC beta-lactamases (Livermore, 1995). In recent years, Metallo-Beta-Lactamase (MBL) genes have spread from Pseudomonas aeruginosa to members of the Enterobacteriaceae. These enzymes are 
plasmid mediated and multidrug resistance is a characteristic feature of strains producing these enzymes (Varaiya et al., 2008a).

The overall prevalence of ESBL, AmpC, MBL and Klebsiella pneumoniae Carbapenemase (KPC) producing Klebsiella pneumoniae are found to vary greatly in different geographical areas and in different institutes of the same area (Coudron et al., 2000).

In our study, $67 \quad(79.7 \%) \quad$ Klebsiella pneumoniae isolates were ESBL producers. In India, the percentage of ESBL producers range from $4 \%$ to $83 \%$ (Rodrigues et al., 2004). Our findings are comparable with the study done by Rampure et al., (2013) who also found $78.21 \%$ of the isolates to be ESBL producers. Increased use of third generation cephalosporins to treat Gram negative infections and monotherapy with cephalosporins may be responsible for this increased resistance (Rodrigues et al., 2004).

ESBL-producing Klebsiella pneumoniae isolates were found to be $100 \%$ sensitive to Imipenem. Similar pattern was observed by Varaiya et al., (2008b).

The current CLSI do not indicate the phenotypic screening and confirmatory tests that should be used for the detection of AmpC $\beta$-lactamases in Klebsiella and Escherichia coli (Coudron et al., 2000).

Several studies have been done on various phenotypic test methods namely the Modified three dimensional test, Modified double disk test, AmpC disk test, Inhibitor based method employing inhibitors like boronic acid, Broth microdilution method and Cefoxitin agar method (Hemalatha et al., 2007).

Singhal et al., (2005) compared Modified three dimensional test and AmpC disk test.
They found concordant result with these methods and concluded that the AmpC disk test was an easier, reliable and rapid method of detection of isolates that harbour AmpC $\beta$ lactamases and can be used in diagnostic laboratories.

However Isoelectric focussing and genotypic characterization are considered as gold standard for the detection of AmpC production (Perez-Perez and Hanson, 2002).

In the present study identified 47 (56\%) isolates as possible AmpC screen positive based on resistance to cefoxitin. Among the 47 screen positive isolates only $36(76.6 \%)$ were confirmed as AmpC producers by AmpC disk test. This indicates that reduced susceptibility to cefoxitin for screening AmpC has poor specificity. Cefoxitin resistance in non-AmpC producers may be due to lack of permeation of porin / porin deficient mutants (Coudron et al., 2000).

In our study, we found $42.8 \%$ of the Klebsiella pneumoniae isolates to be AmpC producers. The prevalence of plasmid mediated AmpC varies widely in different parts of the world from $2 \%$ to $46 \%$ (Coudron et al., 2000).

In the present study observed 31 (36.9\%) Klebsiella pneumoniae isolates as both ESBL and AmpC co-producers. This could be because plasmid mediated AmpC enzymes have also been shown to disseminate among Enterobacteriaceae, sometimes in combination with ESBLs (Singhal et al., 2005). Varghese et al., (2014) found in their study co-existence of ESBL and plasmid mediated AmpC in $4.6 \%$ of Klebsiella pneumoniae whereas a much higher co-existence of $86.1 \%$ was observed by Hemalatha et al., (2007).

Linaresa, et al., (2008) reported that maximum number of $\mathrm{AmpC}$ producing 
isolates were from urine followed by surgical wound infection and observed that the AmpC harbouring isolates were mainly from Intensive Care Unit (ICU), surgical ward and dialysis unit. It is apparent that various mechanisms exist for the production of multiple beta-lactamases specially in high pressure units like Obstetrics and Gynaecology, Surgery and Medicine, where newer beta-lactams are being routinely prescribed (Rodrigues et al., 2004).

AmpC producing Klebsiella pneumoniae isolates were found to be $75 \%$ sensitive to Imipenem. Varghese, et al., (2014) observed in their study that $79 \%$ of the isolates were sensitive to Imipenem, whereas $100 \%$ sensitivity of AmpC producers to Imipenem has been noted by Hemalatha et al., (2007). The available data suggests that carbapenems are more effective than cefepime in treating serious infections that involves large number of AmpC producing organisms. However, we need to keep in mind that carbapenems are antimicrobials that are usually kept in reserve. The cost of Imipenem is a limiting factor for using it in a country like ours (Rodrigues et al., 2004).

Sensitivity to Levofloxacin and Amikacin were $63.9 \%$ and $52.8 \%$ respectively. These drugs can be considered for infections caused by AmpC producing Klebsiella pneumoniae isolates after proper antibiotic susceptibility testing instead of giving empirical carbapenem for therapy. Thus Levofloxacin and Amikacin are cost effective alternative for treating such patients. A shift in empirical therapy to the carbapenems, due to the presence of ESBL producers, is associated with emerging carbapenem resistance in the ESBLproducing organisms (Meyer et al., 1993).

Carbapenem resistance in Klebsiella pneumoniae may be due to the production of
Klebsiella pneumoniae carbapenemase (KPC), combinations of outer-membrane permeability loss and beta-lactamase production, production of metallo-betalactamases (MBL) such as IPM or VIM groups, the New Delhi metallo-betalactamase enzyme (NDM-1), porin OmpK $35 / 36$ insertional inactivation and down regulation of the phosphate transport porin and changes in penicillin binding proteins (Sidjabat et al., 2011) and (Parveen et al., 2010).

Carbapenem resistance is one of the major threats to the antimicrobial treatment of infections with Gram negative organisms. It has emerged among Klebsiella pneumoniae isolates due to the acquisition of carbapenemase, which usually belongs to Ambler class B metallo-beta-lactamases (MBLs) or to Ambler class A KPC-type enzymes (Tsakris et al., 2009).

KPC resistance can co-exist with other Gram negative resistance mechanisms including ESBL, fluoroquinolone and aminoglycoside resistances in addition to carbapenems. The genes for the enzymes are usually on plasmids and therefore can spread readily among members of the same species or even among different genera (Parveen et al., 2010).

According to the CLSI recommendations, Enterobacteriaceae that are resistant to extended spectrum cephalosporins and have a carbapenem MIC $\geq 2 \mu \mathrm{g} / \mathrm{ml}$ or a carbapenem intermediate or resistant zone of inhibition by disk diffusion, may produce a KPC betalactamase. For KPC detection, ertapenem is the most sensitive carbapenem while meropenem and imipenem are more specific than ertapenem.

The definitive detection of a KPC encoding organism can be performed using PCR and /or by Modified Hodge test (Parveen et al., 2010). Despite PCR being highly accurate and 
reliable, its accessibility is often limited to reference laboratories (Walsh et al., 2005).

Several Phenotypic tests have been developed for MBL detection and all these are based upon the ability of chelating agents, EDTA and thiol based compounds, to inhibit MBL activity. Behera et al., (2008) screened for MBL production in their study using Imipenem-EDTA combined disk test, Imipenem-EDTA double-disk synergy test, Imipenem-EDTA MBL E-test and EDTA disk potentiation using four cephalosporins.

They concluded that Imipenem-EDTA combined disk test and Imipenem-EDTA MBL E-test were equally effective for MBL detection and believed that given the costconstraints, Imipenem-EDTA combined disk test could be used as a convenient screening method in the Clinical Microbiology laboratories.

We identified $14(16.6 \%)$ isolates as possible MBL screen positive based on resistance to Imipenem. Among the 14 screen positive isolates, only $12(85.7 \%)$ were confirmed as MBL producers by Imipenem + EDTA Combined disk test. However none of the isolates were positive for Klebsiella pneumoniae Carbapenemase (KPC) by Modified Hodge Test. Parveen et al., (2010) reported $13.3 \%$ Klebsiella pneumoniae isolates to be KPC producers by Modified Hodge Test and found that none of the Klebsiella pneumoniae produced MBL by EDTA-meropenem disk approximation test in their study in South India. Sidjabat et al., (2011) stated in their study that although surveillance studies are lacking, it is known that at least $10 \%$ of Klebsiella pneumoniae in some hospitals in India are NDM producers.

In our study, $12(14.3 \%)$ Klebsiella pneumoniae isolates were ESBL, AmpC and MBL co-producers. Presence of AmpC and / or Cefotaxime (CTX)-M when combined with a loss of porin results in resistance to carbapenems (Varghese et al., 2014).

Table.1 Antibiotic resistance pattern in ESBL, AmpC and MBL-producing isolates

\begin{tabular}{|l|c|c|c|}
\hline Antibiotics & $\begin{array}{c}\text { ESBL positive n } \\
(\mathbf{\%})\end{array}$ & $\begin{array}{c}\text { AmpC positive n } \\
(\mathbf{\%})\end{array}$ & $\begin{array}{c}\text { MBL positive n } \\
(\mathbf{\%})\end{array}$ \\
\hline Ciprofloxacin & $34(50.7 \%)$ & $22(61.1 \%)$ & $7(58.3 \%)$ \\
\hline Levofloxacin & $10(14.9 \%)$ & $13(36.1 \%)$ & $4(33.3 \%)$ \\
\hline Gentamicin & $31(46.2 \%)$ & $23(63.8 \%)$ & $10(83.3 \%)$ \\
\hline Amikacin & $12(17.9 \%)$ & $17(47.2 \%)$ & $8(66.7 \%)$ \\
\hline Cefazolin & $59(88.0 \%)$ & $35(97.2 \%)$ & $12(100 \%)$ \\
\hline Cefoxitin & $20(29.8 \%)$ & $36(100 \%)$ & $12(100 \%)$ \\
\hline Cefotaxime & $67(100 \%)$ & $34(94.4 \%)$ & $12(100 \%)$ \\
\hline Ceftriaxone & $67(100 \%)$ & $34(94.4 \%)$ & $12(100 \%)$ \\
\hline Ceftazidime & $67(100 \%)$ & $34(94.4 \%)$ & $12(100 \%)$ \\
\hline Cefepime & $50(74.6 \%)$ & $25(69.4 \%)$ & $11(91.6 \%)$ \\
\hline Cotrimoxazole & $49(73.1 \%)$ & $26(72.2 \%)$ & $11(91.6 \%)$ \\
\hline Tetracycline & $30(44.7 \%)$ & $19(52.7 \%)$ & $7(58.3 \%)$ \\
\hline Piperacillin+Tazobactam & $1(1.5 \%)$ & $15(41.6 \%)$ & $10(83.3 \%)$ \\
\hline Imipenem & $0(0 \%)$ & $9(25.0 \%)$ & $12(100 \%)$ \\
\hline Ertapenem & $2(2.9 \%)$ & $14(38.9 \%)$ & $12(100 \%)$ \\
\hline
\end{tabular}


Table.2 Risk factors associated with ESBL, AmpC and MBL producing Klebsiella pneumoniae isolates

\begin{tabular}{|c|c|c|c|c|c|c|}
\hline $\begin{array}{c}\text { Risk Factors } \\
\text { (number) }\end{array}$ & $\begin{array}{c}\text { ESBL } \\
\text { positive } \\
\text { isolates } \\
\text { number } \\
(\boldsymbol{\%})\end{array}$ & $\begin{array}{c}\text { P } \\
\text { Value }\end{array}$ & $\begin{array}{c}\text { AmpC } \\
\text { positive } \\
\text { isolates } \\
\text { number } \\
(\boldsymbol{\%})\end{array}$ & P value & $\begin{array}{c}\text { MBL } \\
\text { positive } \\
\text { isolates } \\
\text { number } \\
(\boldsymbol{\%})\end{array}$ & $\begin{array}{c}\text { P } \\
\text { value }\end{array}$ \\
\hline Burns (7) & $6(85.7 \%)$ & $<0.001$ & $3(42.8 \%)$ & $<0.05$ & $1(14.2 \%)$ & $>0.05$ \\
\hline Carcinoma (3) & $2(66.7 \%)$ & $<0.05$ & $2(66.7 \%)$ & $<0.05$ & $0(0 \%)$ & - \\
\hline Chronic ailments (38) & $28(73.6 \%)$ & $<0.0001$ & $14(36.8 \%)$ & $<0.01$ & $2(5.3 \%)$ & $>0.05$ \\
\hline Diabetes mellitus (5) & $5(100 \%)$ & - & $3(60 \%)$ & $<0.01$ & $1(20 \%)$ & $>0.05$ \\
\hline $\begin{array}{c}\text { Intensive care units } \\
(20)\end{array}$ & $17(85 \%)$ & $<0.0001$ & $8(40 \%)$ & $<0.01$ & $2(10 \%)$ & $>0.05$ \\
\hline $\begin{array}{c}\text { Surgical } \\
\text { interventions(78) }\end{array}$ & $60(76.9 \%)$ & $<0.0001$ & $35(44.9 \%)$ & $<0.001$ & $11(14.1 \%)$ & $<0.01$ \\
\hline $\begin{array}{c}\text { Hospitalization for } \\
\text { more than 5 days (60) }\end{array}$ & $51(85 \%)$ & $<0.0001$ & $31(51.6 \%)$ & $<0.001$ & $10(16.7 \%)$ & $<0.01$ \\
\hline
\end{tabular}

MBL producing isolates showed 100\% resistance to $3^{\text {rd }}$ generation cephalosporins (Cefotaxime, Ceftriaxone and Ceftazidime), cefoxitin, cefazolin and carbapenems. High resistance was observed towards Cefepime (91.6\%), Cotrimoxazole (91.6\%) and Gentamicin (83.3\%). Least resistance, $33.3 \%$, was observed towards Levofloxacin. Ciprofloxacin and Tetracycline showed $58.3 \%$ resistance each.

Limbago et al., (2011) stated in their study that although isolates were carrying MBL, they remained susceptible to fluoroquinolones, trimethoprimsulfamethoxazole and tetracyclines. Aminoglycosides, Tigecycline and the Polymyxins are among the few agents available for treating patients who are infected with carbapenemase-producing strains (John Quale, 2008).

All ESBL, AmpC and MBL co-producers showed similar resistance pattern as $\mathrm{MBL}$ producers. Levofloxacin and Amikacin are cost effective alternative for treating such patients. These drugs can be considered for infections caused by MDR Klebsiella pneumoniae isolates after proper antibiotic susceptibility testing instead of giving empirical carbapenem and other reserve drugs for therapy.

\section{Risk factors}

Statistically significant association ( $\mathrm{p}$ value $<0.05$ ) between risk factors and infection with ESBL, AmpC and MBL producing Klebsiella pneumoniae isolates were found in patients with burns, chronic ailments, Diabetes mellitus, hospitalization for more than 5 days, intensive care units and surgical interventions (Table no.2).

Babypadmini and Appalaraju, (2004) reported admission in ICU and surgery to be the risk factors for ESBL production in their study. Pai et al., (2004) observed in their study that long hospital stay, care in intensive care unit, central venous catheterization, indwelling urinary catheter, invasive procedure, neutropenia, post surgical state, nosocomial infection and prior use of antibiotics as significant risk 
factors for the acquisition of AmpC producing Klebsiella pneumoniae isolates.

Daikos et al., (2010) also found prolonged hospitalization, ICU stay, surgery, invasive devices and therapy with multiple antibiotic agents before initial culture as risk factors typically associated with the acquisition of MBL-producing bacteria.

Organisms carrying hidden MBL genes are a threat to infection control efforts and may result in poor clinical outcomes when carbapenems are used for treatment in those with serious infections. The high rate of nosocomial colonization by multidrug resistant Klebsiella pneumoniae appears to be associated with the use of antibiotics rather than with factors connected with delivery of care in the hospital. In the hospital setting, the local antibiotic policy is a major determinant of the colonization pattern (Podschun and Ullmann, 1998).

In conclusion, widespread use of antimicrobials has often been held responsible for the occurrence of multi-drug resistant Klebsiella strains in hospitals. This epidemic of Gram-negative resistance must be stopped. Increased awareness and intensified infection control practices are the keys to curtail the spread of this terrifying antimicrobial resistance.

\section{References}

Babypadmini, S., Appalaraju, B. 2004. Extended spectrum beta-lactamases in the urinary isolates of Escherichia coli and Klebsiella pneumoniae: Prevalence and susceptibility patterns in a tertiary care hospital. Indian $J$. Med. Microbiol., 22(3): 172-174.

Behera, B., et al. 2008. An evaluation of four different phenotypic techniques for detection of metallo-beta-lactamase producing Pseudomonas aeruginosa.
Indian J. Med. Microbiol., 26(3): 233237.

Clinical and Laboratory Standards Institute. 2013. M100-S23. Performance Standards for Antimicrobial Susceptibility Testing; Twenty-Third Informational Supplement.

Coudron, P.E., et al. 2000. Occurrence and Detection of AmpC beta-lactamases among Escherichia coli, Klebsiella pneumoniae and Proteus mirabilis isolates at a Veterans Medical Center. $J$. Clin. Microbiol., 38 (5): 1791-1796.

Daikos, G.L. et al. 2010. Risk factors for bloodstream infection with Klebsiella pneumoniae producing VIM-1 metallo- $\beta$-lactamase. J. Antimicrob, Chemother., 65 (4): 784-788.

Hemalatha, V. et al. 2007. Detection of AmpC beta-lactamase production in Escherichia coli and Klebsiella by an inhibitor based method. Indian J. Med. Res., 126: 220-223.

John Quale. 2008. Global spread of Carbapenemase-producing Klebsiella pneumoniae. Microbe, 3 (11): 516-520.

Lim, K.T. et al. 2009. Characterization of multidrug-resistant and extendedspectrum $\quad \beta$-lactamase-producing Klebsiella pneumoniae strains from Malaysian hospitals. J. Med. Microbiol., 58: 1463-1469.

Limbago, B.M. et al. 2011. IMP-producing Carbapenem-resistant Klebsiella pneumoniae in the United States. J. Clin. Microbiol., 49(12): 4239-4245.

Linaresa, L., et al. 2008. Risk Factors for Infection with Extended-Spectrum and AmpC beta-Lactamase-producing Gram-Negative Rods in Renal Transplantation. Amer. J. Transplantation, 8: 1000-1005.

Livermore, D.M. 1995. Beta-lactamases in laboratory and clinical resistance. Clin. Microbiol. Rev., 8: 557-584.

Longo, Fauci, Kasper, Haucer, Jameson and 
Loscalzo. Diseases caused by Gramnegative enteric bacilli. Harrison's principles of internal medicine. $18^{\text {th }}$ edition.

Meyer, K.S. et al. 1993. Nosocomial outbreak of Klebsiella infection resistant to late-generation cephalosporins. Ann. Intern. Med., 119 (5): 353-358.

Pai, H., et al. 2004. Epidemiology and Clinical Features of Bloodstream Infections Caused by AmpC-Type- $\beta$ Lactamase-producing Klebsiella pneumoniae. Antimicrob. Agents Chemother., 48 (10): 3720-3728.

Parveen, R.M. et al. 2010. Emerging Carbapenem Resistance among Nosocomial Isolates of Klebsiella pneumoniae in South India. Int. J. Pharma. Bio Sci., 1(2): 1-11.

Perez-Perez, F.J., Hanson, N.D. 2002. Detection of plasmid-mediated AmpC beta-lactamase genes in clinical isolates by using multiplex PCR. J. Clin. Microbiol., 40: 2153-2162.

Podschun, R., Ullmann, U. 1998. Klebsiella spp. as Nosocomial Pathogens: Epidemiology, Taxonomy, Typing Methods and Pathogenicity Factors. Clin. Microbiol. Rev., 10 (4): 589-603.

Rampure, R. et al. 2013. Prevalence of MDR-ESBL producing Klebsiella pneumoniae isolated from clinical samples. J. Microbiol. Biotech. Res., 3(1): 32-39.

Rodrigues, C. et al. 2004. Detection of Beta-lactamases in nosocomial Gram negative clinical isolates. Indian $J$. Med. Microbiol., 24 (4): 247-250.

Sidjabat, H. et al. 2011. Carbapenem Resistance in Klebsiella pneumoniae Due to the New Delhi Metallo-betalactamase. Clin. Infect. Dis., 52 (4): 481484.

Singhal, S. et al. 2005. Evaluation of methods for AmpC beta-lactamase in Gram negative clinical isolates from tertiary care hospitals. Indian $J$. Med. Microbiol., 23(2): 120-124.

Tsakris, A. et al. 2009. Evaluation of Boronic acid disk tests for differentiating KPC-possessing Klebsiella pneumoniae isolates in the clinical laboratory. J. Clin. Microbiol., 2: 362-367.

Varaiya, A. et al. 2008a. Incidence of metallo-beta-lactamase producing Pseudomonas aeruginosa in diabetes and cancer patients. Indian J. Pathol. Microbiol., 51 (2): 200-203.

Varaiya, A.Y. et al. 2008b. Extended spectrum beta-lactamase producing Escherichia coli and Klebsiella pneumoniae in diabetic foot infection. Indian J. Pathol. Microbiol., 51 (3): 370-372.

Varghese, D.S. et al. 2014. Concurrent occurrence of AmpC and Cefotaxime (CTX)-M in clinical isolates of enterobacteriaceae. J. Acad. Clin. Microbiologists, 16(1): 11-16.

Walsh, T.R. et al. 2005. Metallo-betalactamases: the quiet before the storm? Clin. Microbiol. Rev., 18(2): 306-325.

\section{How to cite this article:}

Md. Mudassar Iqbal Lodhi and Shobha D. Nadagir. 2016. Prevalence and Antibiotic Susceptibility Pattern of Multi-drug Resistant Klebsiella pneumoniae in Pus samples from Surgical Ward Patients in a Tertiary Care Hospital in South India. Int.J.Curr.Microbiol.App.Sci. 5(8): 635-643. doi: http://dx.doi.org/10.20546/ijcmas.2016.508.071 